\title{
Cooperation through Coordination in Two Stages
}

\author{
Todd R. Kaplan ${ }^{\mathrm{a}}$ \\ University of Exeter \\ University of Haifa \\ Bradley J. Ruffle ${ }^{\mathrm{b}}$ \\ Wilfrid Laurier University \\ Zeev Shtudiner ${ }^{c}$ \\ Ariel University
}

August 2018

\begin{abstract}
Efficient cooperation often requires coordination, such that exactly one of two players takes an available action. If the decisions whether to pursue the action are made simultaneously, then neither or both may acquiesce leading to an inefficient outcome. However, inefficiency may be reduced if players move sequentially. We test this experimentally by introducing repeated two-stage versions of such a game where the action is individually profitable. In one version, players may wait in the first stage to see what their partner did and then coordinate in the second stage. In another version, sequential decision-making is imposed by assigning one player to move in stage one and the other in stage two. Although there are fewer cooperative decisions in the two-stage treatments, we show that overall subjects coordinate better on efficient cooperation and on avoiding both acquiescing. Yet, only some pairs actually achieve higher profits, while the least cooperative pairs do worse in the two-stage games than their single-stage counterparts. For these, rather than facilitating coordination, the additional stage invites unsuccessful attempts to disguise uncooperative play, which are met with punishment.
\end{abstract}

Keywords: experimental economics, cooperation, efficiency, two-stage games, turntaking, timing of moves.

\section{JEL classification nos.: C90, Z13.}

We thank Sean D'Evelyn, Justin Smith, Ro'i Zultan, an anonymous referee and a co-editor of this journal, Dirk Engelmann, and numerous departmental seminar and conference participants for helpful comments. Tim Miller programmed the experiments. Kaplan thanks the Economic and Social Research Council (grant no. RES-000-22-0370) and Ruffle thanks the Israel Science Foundation (grant no. 776/03) for generous financial support.

a Todd Kaplan, Department of Economics, University of Haifa, Abba Khoushy Ave 199, Haifa, 3498838 Israel, todd@econ.haifa.ac.il

b Corresponding Author: Bradley Ruffle, Department of Economics, Wilfrid Laurier University, 75 University Ave. W., Waterloo, ON, N2L 3C5 Canada, bruffle@wlu.ca.

c Ze'ev Shtudiner, Department of Economics and Business Administration, Ariel University, Ariel 40700, Israel, zeevs@ariel.ac.il. 


\section{Introduction}

Sometimes cooperation can be achieved only through coordination. Yet, efficient coordination often requires that players rely on their private information. In any situation in which decisions must be made simultaneously, coordination cannot fully exploit such information. However, if decisions are made over time, then those attempting to coordinate have the ability to make use of their private information. In this paper, we ask whether this extra ability indeed results in increased payoffs.

Consider a quiz show for which a team must put forth a representative to answer a question. All team members have the same objective - success of the team. In this pristine situation, the players need only worry about coordination. For the highest chance of the team being correct, it would want the player with the highest degree of confidence to answer the question (confidence being private information). One selection method is to have players press a buzzer to indicate their willingness to answer. With only a second to buzz, a player can infer little about his teammates' confidence. With more time permitted to buzz, a player can wait and infer that a teammate is less confident if he has not already responded. Thus, more time should increase the chance that a higher-ability player gets to answer the question.

Another way of selecting a player is to ask each sequentially. A player may decline if he thinks there is a better player to follow who would agree. In this case players do not need to infer about other players' information who preceded them. The mechanism itself takes advantage of the private information. A player asked second would effectively make the decision contingent on the first player's private information since that player should not volunteer with a low confidence level.

Both these cases point to the possibility that altering the timing of such decisions can improve coordination. This also applies when players' preferences are not aligned as in most real-world situations, that is, when they are playing a non-cooperative game. We seek to determine whether the increased capability to coordinate helps, using a two-player entry game with congestion first introduced in Kaplan and Ruffle (2012). ${ }^{1}$ Here, a single

\footnotetext{
${ }^{1}$ In that paper, the authors explore several single-stage variations of the above game with the goal of determining whether cooperative behavior takes the form of cutoff strategies (enter on high integers, exit on low ones) or alternating (players take turns entering and exiting).
} 
entrant yields a higher social surplus than if both enter or, worse yet, neither does. The players have private information about their value of being an entrant. One interesting feature of this congestion game is that it sometimes requires cooperative players to act against their short-term interest; that is, they sometimes have to exit despite entry being the dominant strategy of the one-shot game. This tension, which does not exist in pure coordination games, poses a bigger challenge to obtaining cooperation.

We examine three timings of moves, each represented by an experimental treatment in a between-subjects design. ${ }^{2}$ Now: simultaneous decisions. Seq: an exogenous ordering of sequential decisions whereby the first player decides to enter or exit and then, after observing the first player's move, the second player decides to enter or exit. Wait: the players endogenously time their decisions over two stages (similar to a two-period discrete version of the above quiz show example). In addition to entering or exiting in the first stage, each player may choose to wait; namely, he postpones his decision until the second stage giving him the opportunity to observe his partner's first-stage decision, which includes the possibility to wait.

Both the simultaneous game and its dynamic extensions have analogies to numerous real-world cooperation dilemmas. For example, bidders in an auction can actively compete with one another. In so doing, each reduces the other's expected surplus. Or bidders with a sufficiently low value for the good being auctioned can elect not to participate. Alternatively, consider two fast-food chains that each contemplates opening a franchise in a small town. They may possess different expected private values of being the local monopolist that stem from different expected costs or demand for its products. If these two chains wish to collude implicitly, then the chain with a low value would stay out, under the presumption that the favor will be returned in the future. Also, individuals may choose not to enter contests or competitions if their value for the prize or probability of winning is sufficiently low and they care about other more deserving or more capable participants. Junior employees backing down from an internal promotion contest is a common

\footnotetext{
${ }^{2} \mathrm{~A}$ different approach to reducing entry and improving cooperation would be to impose a limit on the total number of entries permitted by each player in the repeated game. In a similar vein, Engelmann and Grimm (2012) examine a two-player voting game where optimal cooperation requires one to vote for their preferred option only when one's private value is high. Interestingly, only when an exogenous budget constraint (in terms of number of votes) is imposed do they observe "cooperation" rather than players pursuing the dominant strategy of exaggerating their values and always voting for their preferred option.
} 
occurrence. Finally, cab drivers, bicycle messengers, golf caddies, waitstaff, sky caps and vendors in a marketplace often face the decision of whether to compete for a customer or acquiesce, with the consequences of their decisions similar to our game's payoff structure.

None of the above-mentioned dilemmas is inherently a simultaneous-move game. For example, a bidder in an English auction might hesitate before calling out a bid to gauge whether other auction participants intend to bid. A firm may postpone the decision whether to enter a market to determine whether a rival firm values the market more as indicated by its swift entry. A cab driver not in the immediate vicinity of the fare may choose to wait to see if other cabbies respond to the dispatcher's call. ${ }^{3}$ In other examples the order of moves may be exogenously given. One bidder may be larger than the others. A chain store may be the market leader. A job, promotion or dating opportunity may be offered first to one candidate who can accept, or decline because he recognizes that the next candidate in line is better suited or more eager.

As suggested by all of these examples, the possibility that players commit at different times to their entry-exit decisions can facilitate a more efficient outcome according to which the player with the higher value for the action pursues it, while the lower-value player acquiesces. To illustrate, if a firm always enters for a certain range of high values, then the possibility of waiting permits the firm to refine its strategy to enter on only a subset of this range and wait otherwise. By waiting and subsequently not entering whenever the other enters, double entry is avoided and a higher social surplus attained. ${ }^{4}$ Similarly, if there exists a natural sequential ordering to the firms' moves, then the second mover can enter whenever the first mover stays out and exit whenever the first mover enters, thereby altogether avoiding double entry and double exit.

In this paper, we show theoretically that permitting non-simultaneity of observable decisions in our game (Wait and Seq) can facilitate cooperation and higher profits

\footnotetext{
${ }^{3}$ Gal-Or (1985) first made the point that the timing of moves (i.e., when to commit) may be a strategic choice variable. See also Dowrick (1986) for a model of quantity competition in which firms choose their roles as leader or follower.

${ }^{4}$ Consider for illustrative purposes the following simplified game parameterization: the set of values is 1,2 and 3, each with an equal chance. If firms enter on a 2 or 3, then double entry occurs $4 / 9$ of the time, no entry $1 / 9$ of the time and single entry the remaining $4 / 9$. By switching to entry on 3 and waiting on 2, double entry occurs only $2 / 9$ of the time (1/9 in the first stage when both have 3 s and another $1 / 9$ in the second stage when both have 2 s). Single entry increases to $2 / 3$ of the time with no entry still at 1/9.
} 
compared to simultaneous moves (Now). We then explore this possibility in a repeated game over 60 rounds with fixed pairings.

Our experiments reveal that Seq has the highest pair profit overall followed by Wait and then Now (but with only Seq significantly higher than Now). However, these findings conceal a deeper picture. On the one hand, cooperative pairs earn much more in the twostage Wait and Seq treatments in which cooperation helps to avoid double exit and coordinate on efficient cooperation. On the other hand, for many others the addition of a second stage reduces cooperation and payoffs. For these low-earning pairs in Wait, the predominant strategy is to wait and then enter regardless of values or the other player's first-stage decision. For the low-earning pairs in Seq, the first mover enters, but rather than acquiesce, the second mover also enters. Thus, while waiting and a second stage enhance outcomes in the hands of cooperative subjects, our experiments also illustrate the potential for these features to lead to worse outcomes. This cannot solely be due to the complexity of coordination in the two stages. In Seq, the second mover needs simply to take the opposite action of the first mover to achieve perfect (though not necessarily efficient) coordination. Notwithstanding, we found that when the first mover enters, the second mover often also enters.

These findings have implications for competition policy and optimal market structure, to be discussed in Section 5. The timing of moves is often a choice variable for a market designer or regulator. However, even when the timing is fixed, an alternation of the timing can often be mimicked by a change in the information structure.

In the next section, we discuss the related literature. Section 3 lays out the experimental design and procedures. Section 4 provides a theoretical framework for our experiments and some testable hypotheses. For each of the experimental treatments, section 5 presents the results, including the degree of cooperation, the pairwise coordination of outcomes, subjects’ profits and an individual strategy analysis. Section 6 concludes with some implications for market design.

\section{Previous Results on Cooperation and the Timing of Moves}

There have been a number of studies that compared sequential to simultaneous moves. In two-player step-level public-goods experiments, Normann and Rau (2015) show that 
sequential moves increase public-good provision and payoffs compared to simultaneous decisions, even though some first-movers contribute less than half of the threshold and are punished by second-movers. In a sequential prisoners’ dilemma game, Clark and Sefton (2001) observe high degrees of reciprocation, with second-movers frequently cooperating in response to first-movers' cooperation, but rarely cooperating after their defection. Sequential moves in our setting can straightforwardly improve outcomes by avoiding double exit and double entry altogether: the second-mover needs to take the opposite action of the first-mover such that exactly one player always enters.

In coordination games with Pareto-ranked equilibria (e.g., weakest link/minimum effort games, van Huyck et al. 1990), players are able to reach the highest-effort equilibria in both sequential and simultaneous-move versions when they are only two players (Harrison and Hirshleifer 1989). While with larger groups, studies find that efficiency is not achieved ${ }^{5}$, presumably sequential choice should at least weakly improve efficiency. When equilibria in coordination games cannot be Pareto ranked, one-shot play of a sequential game should favor the first mover. In repeated play, we would expect to obtain turn-taking, similar to that found in Kaplan and Ruffle (2012).

Our paper also contributes to the literature on the endogenous timing of moves. In Wait, the addition of the wait option renders endogenous the timing of players' decisions to enter or exit. In Cournot duopolies, when the timing of quantity decisions is endogenous, players may postpone their decisions in order to make strategic use of the other player's actions. For example, in Hamilton and Slutsky's (1990) extended game with action commitment, firms can choose their quantities in one of two stages before the market clears. By waiting until period 2, a firm observes the other firm's first-stage action. When the basic duopoly game has a unique equilibrium in the interior of the action space, the authors show that endogenous Stackelberg leadership with one firm choosing its action in stage 1 and the other in stage 2 are the only pure-strategy equilibria. Likewise, when publicly observable decisions reveal agents' private information, the strategic delay of decisions may be an equilibrium (see Chamley and Gale 1994; Gul and Lundholm 1995).

\footnotetext{
${ }^{5}$ See the survey of Devetag and Ortmann 2007. An exception is Engelmann and Normann (2010) in which even groups of six mostly Danish subjects are able to coordinate on the Pareto-efficient equilibrium in the minimum-effort game.
} 
In laboratory experiments, Huck et al. (2002) investigate the duopoly game of Hamilton and Slutsky with symmetric firms. By giving players the option of moving early or late they allow quantity choices to be made either simultaneously (Cournot) or sequentially (Stackelberg). Their data do not support the theory: Stackelberg outcomes are extremely rare. In the majority of cases both players choose to move early and make their choices simultaneously. Moreover, coordination failures occur in $25 \%$ of all cases.

In complementary papers, Nosenzo and Sefton (2011) and Gächter et al. (2010) also compare experimentally a two-period, endogenous-move game to both a two-period, sequential-move game and a one-period, simultaneous-move game. This was done using a public-good game first analyzed by Varian (1994).

Two key design differences from our experiment are that these experiments were done with random matching rather than fixed pairs, making a cooperative outcome more difficult to obtain, and with complete information, which eliminates any cooperative advantage to delaying in such games. Despite these differences, their results do share common elements with ours. In particular, in the sequential format, a free-riding first-mover is often punished by a second-mover leading to more cooperative behavior. However, unlike in our experiments, this potential punishment seems to cause both players to wait in their endogenous treatment leading to identical results to those obtained in their simultaneous game.

Informational asymmetries between players provides another motivation to study sequential versus simultaneous moves. For example, sequential moves permit better informed players to move first, thereby allowing them to signal their private information to less informed followers who may imitate their actions. Potters et al. (2005) examine a two-player public-goods game in which one player is better informed than the other about the value of the public good. In one treatment, they allow players to vote on a simultaneousor sequential-move structure. They find that $81 \%$ of the pair-round decisions results in sequential moves and that the resulting contributions are higher than in the simultaneousmove games. Ziegelmeyer et al. (2005) study the timing of moves in an endogenous waiting game in which two agents receive private signals about an asset with a commonvalue component. Although costly in their experiments, waiting allows players to learn about others' private signals through their choice of action. As predicted, better informed 
players tend to move first and the less-informed followers rationally imitate their investment decision. In our setup, players differ not in their information about the quality of the good or investment, but rather in the strength of their preferences.

Our paper is the first to study simultaneous- versus sequential-move games in an environment in which players have private information about their payoffs. Moreover, the direction and magnitude of the ex-post payoff asymmetry changes from one round to the next and is unknown to players, thereby making coordination on cooperative outcomes especially challenging.

\section{Experimental Design and Procedures}

\subsection{Underlying Game and Treatments}

Our underlying single-stage game is as follows: each subject in the pair privately receives an independently and randomly drawn integer between 1 and 5. In the baseline treatment Now, each subject simultaneously decides whether to enter or exit. The decision to exit yields a payoff of 0 , whereas entry yields the value of the number if the partner exits and $1 / 3$ of the value of the number if the partner also enters. This game is repeated over 60 rounds. Subjects observe their partner's decision and value at the end of each round. The other two treatments, Wait and Seq, extend this single-stage game to permit a second stage.

In Wait each subject decides simultaneously in stage 1 whether to enter, exit or wait. Waiting in stage 1 allows the subject to observe his partner's stage-one decision (but not value) before deciding in stage 2 whether to enter or exit. Waiting is costless; the payoffs depend only on the players' final decisions to enter or exit. Thus, the payoff structure is identical to that in Now.

In Seq a sequential ordering of moves is imposed. One player is randomly assigned to the role of choosing between enter and exit in stage 1 (Player 1). The other player (Player 2) observes Player 1's stage-one decision (but not value) and decides in stage 2 whether to enter or exit. These roles are fixed across all 60 rounds. Again, the payoff structure in Seq is identical to that of the other two treatments. 


\subsection{Experimental Procedures}

We employed a between-subjects design such that participation was limited to a single session and treatment. Upon arrival, all subjects were handed the instructions (see the Online Supplement). After reading them by themselves, the experimenter read them aloud. To ensure that the game was fully understood, subjects answered a series of test questions about the game. Participation in the experiment was contingent upon correctly answering all of the questions, which everyone did. The experiments were conducted in z-Tree (Fischbacher 2007). Before the actual game began, five practice rounds were conducted with identical rules to enable subjects to understand the rules, experiment with different strategies and figure out their preferred strategy before play of the paid rounds. To reduce the possible influence of the five practice rounds on the paid rounds, subjects were rematched with a different partner for the paid 60-round experiment.

Before beginning the sessions, we drew two random sequences of 65 values (for the 60-round game and 5 practice rounds), one sequence for each pair member. We used these sequences for all pairs in all sessions and treatments. This eliminates the need to account for the random variation in values across pairs and treatments and allows us to compare more cleanly the subject pairs' decisions.

The subjects were students at Ben-Gurion University. Eighty-six subjects (43 fixed pairs) participated in Now, 88 subjects (44 fixed pairs) participated in Wait and 80 subjects (40 pairs) in Seq. A Now session lasted about 90 minutes on average, while the Wait and Seq sessions each lasted about 120 minutes. Subjects' profits were converted to shekels at a fixed experimental-currency-to-shekel ratio of 1:0.9. Subjects earned approximately 75 shekels on average (about \$21 USD).

\section{Theoretical Framework and Hypotheses}

\subsection{Theoretical Framework}

The theoretical framework and properties of the single-stage game are presented in Kaplan and Ruffle (2012). There are non-cooperative and cooperative solutions to this game. The Bayes-Nash equilibrium is to follow the dominant strategy of always entering, which yields 
a joint expected payoff of 2. One cooperative solution is for one player to enter and the other to exit. In a repeated game, this cooperative solution can take the form of players taking turns entering and exiting. ${ }^{6}$ The pair's expected payoff from playing the alternating strategy is 3 . Another cooperative solution is to enter only with high numbers, such as 3,4 and 5. This cutoff strategy yields a slightly lower joint expected payoff of 2.88. Notwithstanding, Kaplan and Ruffle (2012) find it to be the modal strategy in their design, which corresponds here to the Now treatment.

In Wait, a stage-one strategy maps values into the possible actions of enter, exit or wait. Full cooperation (maximizing a pair's joint profits) entails monotonic stage-one strategies. Namely, if the action for value $\mathrm{x}$ is enter, then the action for all values $\mathrm{v}>\mathrm{x}$ is also enter. Also, if the action for value $\mathrm{x}$ is wait, then the action for all values $\mathrm{v}>\mathrm{x}$ is either wait or enter (see the Appendix for the proof). It is worth noting that, in contrast to Now in which alternating is the joint-payoff-maximizing strategy, turn taking in stage 1 can never be part of the social optimum in Wait (see the last paragraph of Appendix for the proof).

Table 1 displays the joint expected payoffs for all possible pairings of the 21 monotonic strategies and alternating. To describe the monotonic strategies, we use the following notation: the player exits with values to the left of the parentheses, waits with values between the parentheses, and enters with values to the right of the parentheses. For example, a player who follows the strategy 12(34)5 exits when he receives a value of 1 or 2 , waits when he receives a 3 or 4 , and enters on a 5 . The expected payoff calculations assume that players play cooperatively in the second stage. Namely, if a player waits in the first stage, he enters in the second stage if the other player exited in the first stage and exits if the other player entered in the first stage. If both players chose to wait in the first stage, they employ the alternating strategy to resolve which one enters in stage two. ${ }^{7}$

[Table 1]

\footnotetext{
${ }^{6}$ In addition to Kaplan and Ruffle (2012), turn taking has been observed in Kwasnica and Sherstyuk (2007) in the form of bid rotation in multi-object auctions with complementarities between the objects, in Helbing et al. (2005) to resolve traffic congestion problems in a route-choice game, as well as in Zillante (2011), Cason et al. (2012), Sibly et al. (2018) and Bjedov et al. (2016).

${ }^{7}$ Other payoff-inferior, second-stage strategies exist. For example, with the first-stage strategy $1(234) 5$, one second-stage strategy involves exiting on a value of 2 and entering on values 3 and 4 , if the other player also waited in stage one. This strategy, written as $1(2 / 34) 5$, yields a joint expected payoff of 3.43 , while the strategy $1(23 / 4) 5$ yields a joint expected payoff of 3.20 .
} 
Table 1 shows that several pairs of strategies (and obviously the reverse combinations) achieve the highest joint expected profit of 3.60: 123()45-(12345), 12(3)45-(12345), 12()345-(12345), where the dash separates player 1's strategy from player 2's. This profit compares favorably with the full-information first-best expected surplus (i.e., only the player with the higher value enters) of 3.80. The first strategy pair above divides the expected profit evenly between pair members. Nonetheless, because all three of the above strategy pairs are asymmetric, we anticipate difficulty coordinating on them. Symmetric strategies are more likely to emerge. From the diagonal in Table 1, the most profitable symmetric strategies are 1(234)5 and 1(23)45 with joint expected profits of 3.53 and 3.44, respectively.

From the previous paragraph, we saw that all three of the asymmetric joint-profitmaximizing strategies in Wait require one player to always wait in stage one, regardless of the player's value, and select between enter and exit only in stage two. The Seq treatment precludes Player 1 from waiting while imposing it on Player 2. Thus, the two payoffmaximizing pairs of strategies in Wait that never require Player 1 to wait are also associated with the highest expected payoffs in Seq.

\subsection{Hypotheses}

As the previous subsection illustrates, both the Wait and Seq treatments offer the potential to better coordinate on efficient cooperation and to avoid double entry and double exit. In Now, the cooperative pair can obtain a maximum joint expected payoff of 3 by taking turns entering and exiting. However, Kaplan and Ruffle (2012) show that few subjects are able to coordinate on taking turns. Instead, cooperators play cutoff strategies. In this game, the socially optimal cutoff strategies of 12()345 yield a joint expected payoff of 2.88. If paired subjects play this strategy, double entry occurs with probability $9 / 25$, double exit with probability 4/25 and efficient coordination the remaining 12/25.

By comparison, we anticipate cooperative subjects in Wait to adopt symmetric strategies that involve waiting. The socially optimal symmetric strategies of 1(234)5 and 1(23)45 yield joint expected profits of 3.53 and 3.44, respectively. Both reduce the probability of double exit to $1 / 25$ and of double entry to $1 / 25$ for the former strategy and to $4 / 25$ for the latter one. The result is that these strategies achieve roughly $20 \%$ higher profits 
than the most profitable strategies in Now by committing to a first-stage entry or exit decision for fewer values than in the single-stage game. In this way, if only one player enters or exits in stage 1, his cooperative partner who waited simply chooses the opposite action in stage 2.

Cooperative subject pairs in Seq avoid double entry and double exit altogether. Whatever Player 1's choice of action in the first stage, a cooperative Player 2 chooses the opposite action to ensure precisely one pair member enters. Consequently, profits are expected to be highest in Seq. A cooperative pair earns 3.60 in expectation by playing either of the following strategy pairs: 123()45-(12345), 12()345-(12345). These strategies differ only in Player 1's choice of action when he draws a value of 3. Both strategy pairs lead to efficient cooperation with probability $21 / 25$ and inefficient cooperation with the remaining 4/25 probability.

For each treatment, the left column in Table 2 summarizes these predictions. In brief, the ability to postpone the entry decision in the Wait treatment ought to reduce double entry and double exit and facilitate efficient coordination. To the extent that subjects behave cooperatively, the staggered timing of decisions in Seq ought to eliminate entirely double entry and exit. Consequently, we expect profits to be highest in Seq followed by Wait and lowest in Now.

[Table 2]

\section{Results}

\subsection{Entry}

Surprisingly and counter to our conjecture, a comparison of treatments according to the overall percentage of entry (see the left panel of Table 3) reveals a higher percentage of entry decisions in Wait (77.2\%) and Seq (76.6\%) than in Now (71.9\%). Higher entry on values 1 and 2 in the two-stage games accounts for the higher entry overall in these treatments. Specifically, subjects are 17 and 19 percentage points (hereafter “p.p.”) more likely to enter on a 1 in Wait (38.0\%) and in Seq (39.9\%), respectively, than in Now (20.6\%). This disparity in entry frequency between treatments is of a similar magnitude for the value 2 . Are subjects beating up one another in the two-stage games or do these higher 
entry percentages attest to the successful avoidance of double exit? We will answer this question in the next subsection.

[Table 3]

For the value 3, the disparity reverses: subjects in Now enter 10 p.p. and six p.p. more frequently than their counterparts in Wait and Seq, respectively. Because entry percentages approach $100 \%$ in all three treatments for values 4 and 5, differences between treatments become negligible. If we treat each pair of subjects’ fractions of decisions corresponding to double entry, single entry and no entry over all 60 rounds as the unit of observation, then the non-parametric Kruskal-Wallis test rejects the equality of the entry frequency distributions at the $10 \%$ level $\left(\chi^{2}=4.74, p=.094, n=126\right)$.

\subsection{Coordination of Outcomes}

Despite significantly higher levels of entry in the two-stage games, Table 2 shows that paired subjects in these Wait and Seq treatments managed to avoid double exit and coordinate more frequently on the efficient-cooperation outcome whereby only the player with the higher value entered. More precisely, inefficient double exit drops from $8.7 \%$ in Now to $2.8 \%$ in Wait and to a negligible $0.6 \%$ in Seq. At the same time, efficient cooperation increases by 2 p.p. and 5 p.p. in Wait and Seq compared to the simultaneousmove treatment. However, double entry - the most frequent outcome in all three treatments - displays a modest increase in the non-simultaneous games, rising from $52.4 \%$ in Now to $57.1 \%$ in Wait and $53.8 \%$ in Seq.

The center and right panels of Table 3 display the distributions of stage-one decisions in Wait and Seq, respectively, for each of the five values. In both treatments and in Now (left panel), exit is the modal decision for value 1. Exit remains the modal decision for value 2 in Now and Seq only. In Wait, the wait option becomes the modal choice for values 2 and 3. Yet, the fact that overall entry percentages are marginally higher in Wait than in Seq suggests that many stage-one wait decisions convert into stage-two entry decisions.

Table 4 displays the stage-two entry percentages in each two-stage treatment conditional on the subject's value and the partner's stage-one decision. Subjects have no difficulty entering when their partner exited in stage 1: in both treatments, entry percentages are close to or at $100 \%$ for all values conditional on the partner having exited 
in stage 1. In Wait, after observing the partner's stage-one entry, subjects typically exit on values 1 and 2 if their partner entered; exit remains the modal decision (44\% entry) on a 3. However, the entry percentage jumps to $80 \%$ and $96 \%$ on values 4 and 5 . If the partner also waited in stage 1 , entry is the modal decision on all five values and reaches $100 \%$ for values 4 and 5 . Thus, having received a 4 or a 5 and having chosen to wait in stage 1 , the table shows that entry is almost certain in stage 2 . This begs the question why subjects with values 4 and 5 bothered to wait in stage 1 if their intention was to enter in stage 2 independent of their partner's first-stage decision.

In Seq, after observing one's partner enter in stage 1, the subject also enters in the second stage $82 \%, 92 \%$ and $96 \%$ of the time with values 3, 4 and 5, respectively. With such high entry frequencies, it is no wonder double entry accounts for over half of the outcomes in this treatment.

[Table 4]

\subsection{Profits}

From a theoretical point of view, as section 4.1 illustrates, both the Wait and Seq treatments offer the potential to better coordinate on efficient cooperation. Yet, behaviorally, the additional stage may invite uncooperative play and allows for punishment. This duality can be seen in our results. On the one hand, overall higher entry in Wait and Seq would lead us to expect lower profits than in Now. On the other hand, we saw in the previous subsection that subjects in the two-stage games avoid double exit and succeed in attaining efficient cooperation more often. Table 5 displays summary statistics for paired players' profits. It turns out that pair profits are highest in Seq (170.2 on average), followed by Wait (167.9) and lowest in Now (163.2). If we take each pair's final cumulative profit as the unit of observation and compare the distribution of pair profits for any two treatments, the ranksum Mann-Whitney test reveals only one significant difference: final pair profits in Seq are significantly higher than those in Now $(\mathrm{z}=-2.04, \mathrm{p}=0.04)$.

[Table 5]

The more striking contrast concerns the differential degrees of dispersion of paired subjects' profits across treatments. To begin, note from column 3 of Table 5 that the standard deviation of pair profits of 13.1 in Now is about half that of the Wait and Seq 
treatments. The histograms of pair profits in Figure 1 offer some insight. In Now, fully 40 out of 43 subject pairs' profits are concentrated between 140 and 180. Moreover, 32/40 pairs earn in the narrower range of 160 to 180 . By contrast, the pair profit distributions for the Wait and Seq treatments resemble a uniform distribution over the entire range of 120 to 220. In fact, the Pearson $\chi^{2}$ test cannot reject that the observed pair profit distributions are drawn from a uniform distribution between 120 and 220 in 10 -unit bins $\left(\chi^{2}(9)=8.06\right.$, $\mathrm{p}=.53$ and $\chi^{2}(9)=5.23, \mathrm{p}=.81$ for the Wait and Seq treatments, respectively).

It appears that the two-stage games permit cooperative pairs to do better than is possible in the single-stage game, whereas uncooperative pairs tend to do worse with the addition of the second stage. Why would the better conditions for coordination in Wait and Seq affect pairs in different directions? To address this question, we analyze in the next two subsections how the behavior of the low-profit pairs in Wait and Seq differs from that of their high-profit counterparts.

[Figure 1]

\subsection{Individual Strategy Inference}

Recall from Section 4 that in the Wait treatment there are 21 possible monotonic cutoff strategies in stage 1 that condition on the subject's value. For each subject we compare the ability of each of the 21 monotonic cutoff strategies in Table 1 and the alternating strategy to classify correctly subjects' stage-one decisions. The strategy that minimizes the number of errors in classifying the subject's observed decisions is deemed the strategy the subject most likely employed. Table 6 presents the distribution of these best-fit strategies for stage 1 of Wait. $^{8}$ For each strategy we denote the number of subjects that employ the strategy (column 2), ${ }^{9}$ the mean fraction of errors (deviations from the strategy) by those who employed it (column 3), the mean profit of those who employed the strategy and that of those subjects’ partners as well as the mean entry percentage (after both stages of play) for the subject and his partner.

\footnotetext{
8 The inferred strategies are based on rounds 6-55 to exclude learning in the initial rounds and observed endgame effects. The distributions of best-fit strategies are highly similar for other ranges of included periods, such as all 60 rounds, the first 50 or 55 rounds and the last 50 or 55 rounds.

${ }^{9}$ For several subjects, two or more strategies tied for the fewest errors. In these cases, we assign a share of $1 / n$ to each of the $n$ tied strategies.
} 
[Table 6]

Seventy-five out of the 88 subjects (85\%) employ strategies that involve waiting. The remaining subjects whose strategy does not include waiting either enter on all values (9\%), exit on 1 and enter on values 2-5 (6\%), or exit on 1 and 2 and enter on values 3-5 (1\%). Capturing 25/88 subjects, ${ }^{10}$ the strategy $1(23) 45$ is the most widely employed and the second most profitable strategy among those played by subjects, as evidenced by the high realized mean profit of 95.0 (column 4 of Table 6). For pairs in which at least one subject followed the strategy 1(23)45, pair members earned similarly high profits (see column 5 of Table 6), implying a high degree of reciprocal cooperation. In fact, 22 subjects who played this strategy were paired with one another (11 pairs). Only three subjects were paired with a subject who played a different strategy. Those who followed the strategy 1(23)45 also recorded comparatively few deviations from their inferred strategy. ${ }^{11}$ Tracking this strategy to stage 2, subjects on the whole appear to be playing the strategy 1(2/3)45 (wait with values 2 and 3 and, if the partner also waited, exit on a 2 and enter on a 3).

The strategy of (123)45 is the second most widely used strategy with 14/88 subjects using it, 12 as their sole strategy and two subjects tied with one or more other strategies. While the 1(23)45 and (123)45 strategies differ only in the subject's decision to exit or wait, respectively, on the value of 1 , those who played the latter strategy are paired with even less cooperative partners - ten subjects paired with (123)45 played (12)345. This results in substantially lower mean profits of 82.3 for the subject and 79.9 for the partner compared to those observed for the 1(23)45 strategy. Consistent with this result is the observation that among those who play (123)45, the modal stage-two choice is to enter on

\footnotetext{
${ }^{10}$ We inferred this strategy as the unique best-fit strategy for 23 subjects. For two additional subjects, this strategy tied with one other for the fewest number of errors.

${ }^{11}$ Overall, the error rates are low for most strategies, thereby attesting to the effectiveness of this simple technique in capturing subjects' behavior. Of the 4400 decisions made by the 88 subjects in Wait and the 4000 decisions made by the 80 subjects in Seq between rounds 6-55, 3740 (or 85\%) and 3615 (or 90\%) correspond to the best-fit strategy inferred for each subject compared to 4009 out of the 4300 (or 93\%) decisions made by the 86 subjects in Now. The addition of the waiting option in Wait increases the number of monotonic pure-strategy cutoffs from six in Now to 21 in Wait, thus accounting for the highest error rate in Wait and lowest in the single-stage, binary-choice game, Now.
} 
all values, conditional on the other player having waited (and for some players, even when the other player entered in the first stage).

One lone subject employed the joint profit-maximizing symmetric strategy of 1(234)5, while no pair was found to play any of the asymmetric strategy pairs that earn more than 1(234)5. Nor did any pairs in Wait adopt the payoff-inferior alternating strategy.

Another 5.5 subjects played the strategy (12345), namely, “always wait”. Looking at the second-to-last column of Table 6, we see that these six subjects entered an astonishing $98 \%$ of the time in stage 2, eight p.p. higher than the "always enter" subjects in Wait and 9 p.p. higher than the "always enter" subjects in Now! That is, the always-wait subjects are wholly uncooperative - even more so than those whose best-fit strategy is always enter. The availability of the waiting option seems to attract the least cooperative types; they exercise this option in stage one, only to enter in stage two.

Now the natural question to ask is: were they successful in their attempt to deceive their partners? The last column of Table 7 reveals that their uncooperativeness was reciprocated with entry of $90 \%$ by their partners, slightly higher than the $86 \%$ entry by partners paired against “always enter” in Wait and 85\% against “always enter” in Now. Hence, subjects' attempt to deceive was foiled, leading to low profits for themselves and the pair overall. Always waiting in stage one followed by stage-two entry failed to conceal uncooperativeness.

\section{[Table 7]}

In Table 7 we group together the best-fit strategies for subjects in Now (left panel) as well as for Player 1 subjects in Seq (right panel). The strategy 12()345 according to which subjects exit on values 1 and 2 and enter on values 3, 4 and 5 is the predominant best-fit strategy in Now (53/86 or 62\% of subjects) and among Player 1 subjects in Seq (20/40 or $50 \%)$. In striking contrast, only a single subject utilized this strategy in Wait (1/88) in which the waiting option is also available. Only two pairs of subjects used the alternating strategy in Now despite it being the most profitable strategy in this treatment.

Subjects who played the dominant strategy of ()12345 (i.e., always enter) in Now earned a profit of 74 on average, 14\% less than the mean profit earned by subjects who played the socially optimal cutoff strategy 12()345. In the non-simultaneous-move treatments, by contrast, subjects who played always enter earned only 67.7 and 65.0 in 
Wait and Seq, respectively, which are between $40 \%$ and $48 \%$ below the mean profit of 95 for the socially optimally symmetric cutoff strategy of 1(23)45 in Wait and the mean profit of 96.4 from Player 1's socially optimally cutoff strategy of 123()45 in Seq. The message is that cooperative subjects earn substantially more than uncooperative ones, especially in the Wait and Seq treatments in which cooperation helps to avoid double exit and coordinate on efficient cooperation.

\subsection{Behavior of High- and Low-Profit Subjects in Wait and Seq}

We saw that inefficient cooperation and double exiting are uncommon in Wait (accounting for $1.7 \%$ and $2.8 \%$ of the total outcomes respectively according to Table 3) and in Seq (3.8\% and $0.6 \%$, respectively). Thus, the source of low profits in these treatments must be double entry. If players fully cooperate with one another, double entry would occur in only $4 \%$ of the rounds in Wait and would be entirely absent (0\%) in Seq. Instead, it accounts for $57 \%$ and $54 \%$ of the outcomes in the respective two-stage treatments (see Table 3), a testament to low levels of cooperation among some pairs.

By the structure of the games, double entry must take place in stage 1 in Now and in staggered stages in Seq, whereas in Wait it can be the result of both subjects entering in stage 1 , both waiting in stage 1 and entering in stage 2 , or one entering in stage 1 and the other in stage 2. In fact, $57 \%$ of instances of double entry in Wait arise from both subjects entering in stage $1,17 \%$ from both entering in stage 2 , and a troubling $26 \%$ from subjects entering in different stages, attesting to uncooperative decisions.

We already observed from Table 4 subjects' tendency to largely disregard their partners' first-stage decision and enter on values 4 and 5, and also on value 3 if the partner also waited. The two graphs with triangle markers in Figure 2 display the stage-two entry frequencies conditional on the paired partner waiting in stage 1 in the Wait treatment for the nine lowest profit pairs (earnings between 120 and 139) and the six highest profit pairs (earnings between 200 and 220). ${ }^{12}$ Many of the low-profit subjects chose to wait in stage 1 regardless of their value. Moreover, the figure reveals that all of the entry percentages

\footnotetext{
${ }^{12}$ Table C.9 in the online supplement examines more closely waiting subjects' stage-2 choices conditional on their partner also having chosen to wait in stage 1 as a function of their values and their pairs' realized profits. Table C.10 is an analogous table conditional on the partner having chosen to enter in stage 1.
} 
for these subjects exceed $83 \%$, rising to $100 \%$ for values 4 and 5 . On the other hand, the paucity of observations for values 1, 4 and 5 for the highest-profit pairs (see Table C.9) reflects a more selective use of the waiting option. The six highest-earning pairs almost always exited on a 1 in stage 1 and entered on a 4 and 5. They primarily invoked the wait option when they received values 2 and 3.

[Figure 2]

The differences in entry percentages across profit categories for a given value are even more stark conditional on the partner entering in stage 1 (see the two graphs with the circle markers in Figure 2 and Table C.10 in the online supplement). Despite the partner having already visibly entered in stage 1, paired subjects in the lowest-profit category nonetheless enter with a frequency of $68 \%$ on a value of 1 , increasing to $100 \%$ on values 4 and 5 . Contrastingly, the highest-profit subjects never entered on a value of 1 or 2 and their entry frequency increases to only $11.1 \%$ on a 4.

In summary, successful subject pairs condition their second-stage entry decision on their value and their partner's first-stage decision, whereas lower profit pairs tend to disregard both of these and enter in stage two.

The dissimilarity in stage-two behavior between low-profit and high-profit pairs is equally striking in the Seq treatment. Having observed their partners (Player 1) enter in stage 1, the two graphs with the plus (“+”) markers in Figure 2 as well as Table C.11 in the online supplement reveal that Player 2 subjects from the lowest-profit pairs nonetheless enter $3 / 4$ of the time even on a 1, quickly increasing to $100 \%$ entry on values 3,4 and 5 . By contrast, Player 2 subjects in the highest-profit pairs never enter on a $1(0 \%)$, with their entry frequency rising gradually to $36 \%$ on a value of 4 and $64 \%$ on a value of 5 .

\section{Conclusions}

When collusion in entry games involves avoiding congestion, theory suggests that permitting non-simultaneity of observable decisions is helpful. We find that in practice, only some pairs take advantage of this opportunity, while for many non-simultaneity of moves leads to worse outcomes.

At first glance, converting a single-stage game, Now, into two-stage games, Wait and Seq, increases the frequency of individual uncooperative decisions. Yet the timing of 
these decisions enabled cooperative pairs to achieve a higher degree of pairwise cooperation and substantially higher profits in these two-stage games. However, a closer look at the distribution of profits reveals fully half of the subject pairs in Wait and 35\% of the subject pairs in Seq earned less than the average earnings in Now. The dark side of the waiting option is that this cooperation-enabling tool can be abused by selfish individuals to disguise their uncooperative behavior. Rather than conspicuously entering right away, waiting veils their intent. Similarly, most first movers in Seq enter more than is socially optimal, perhaps feigning higher values. Second movers retaliate by entering even when it causes congestion. In Seq, first movers can mask their selfishness (entering with low values) with a potential for genuine need (having a high value).

Our paper illustrates how an institutional change can have important consequences for cooperation and efficiency. Hence, our results can yield advice for those (re)designing institutions. Take for example a company or government involved in the procurement of a large contract (e.g., construction project, fighter jet) or selling off a large asset (e.g., crown corporation, real estate or spectrum). It is typical to conduct one of two auction-type procedures. One procedure involves a single stage in which bidders enter and submit bids. A second procedure is to hold a two-stage process in which potential entrants are made known in the first stage and bidding occurs in the second stage. Intuitively, one would think that in order to prevent collusion a designer would want less information revealed between bidders and thus favor one stage over two stages or if there are two stages to choose not to publicize the bidders participating. However, if the designer is primarily concerned with attracting at least one bidder, then a two-stage design should be modified to involve sequentially asking bidders in the first stage if they wish to participate and announcing entry results in the second stage. This advice is based on our finding that double exit is lowest in Seq.

If the designer is primarily concerned with instigating a competitive process, then the standard two-stage procedure should be used based on our finding that Wait has the most double entry. Finally, if the designer is primarily concerned with inducing the lowcost supplier to enter, then either non-simultaneous-move process will suffice based on our finding that Seq and Wait have similarly high levels of both efficient cooperation and double entry. 


\section{References}

Bjedov, T., Madiès, T., Villeval, M.C., 2016. Communication and coordination in a twostage game. Economic Inquiry 54(3), 1519-1540.

Cason, T.N., Lau, SH.P., Mui, VL. 2012. Learning, teaching, and turn-taking in the repeated assignment game. Economic Theory 54(2), 335-357.

Chamley, C., Gale, D., 1994. Information revelation and strategic delay in a model of investment. Econometrica 62, 1065-1085.

Clark, K., Sefton, M., 2001. The sequential prisoner's dilemma: evidence on reciprocation. Economic Journal 111(468), 51-68.

Devetag, G., Ortmann, A., 2007. When and why? A critical survey on coordination failure in the laboratory. Experimental Economics 10(3), 331-344.

Dowrick, S., 1986. von Stackelberg and Cournot duopoly: choosing roles. RAND Journal of Economics 17(2), 251-260.

Engelmann, D., Grimm, V., 2012. Mechanisms for efficient voting with private information about preferences. Economic Journal 122(563), 1010-1041.

Engelmann, D., Normann, HT. 2010. Maximum effort in the minimum-effort game. Experimental Economics 13(3), 249-259.

Fischbacher, U., 2007. z-Tree: Zurich toolbox for ready-made economic experiments. Experimental Economics 10(2), 171-178.

Gächter, S., Nosenzo, D., Renner, E., Sefton, M., 2010. Sequential vs. simultaneous contributions to public goods: Experimental evidence. Journal of Public Economics 94(7), 515-522.

Gal-Or, E., 1985. First mover and second mover advantages. International Economic Review 26(3), 649-653.

Gul, F., Lundholm, R., 1995. Endogenous timing and the clustering of agents' decisions. Journal of Political Economy 103, 1039-1066.

Hamilton, J.H., Slutsky, S.M., 1990. Endogenous timing in duopoly games: Stackelberg or Cournot equilibria. Games and Economic Behavior 2, 29-46. 
Harrison, G.W., Hirshleifer, J., 1989. An experimental evaluation of weakest link/best shot models of public goods. Journal of Political Economy 97(1), 201-225.

Helbing, D., Schonhof, M., Stark HU., Holyst, J.A., 2005. How individuals learn to take turns: emergence of alternating cooperation in a congestion game and the prisoner's dilemma. Advances in Complex Systems 8(1), 87-116.

Huck, S., Muller, W., Normann, HT., 2002. To commit or not to commit: endogenous timing in experimental duopoly. Games and Economic Behavior 38, 240-264.

Kaplan, T., Ruffle, B.J., 2012. Which way to cooperate. Economic Journal 122(563), 1042-1068.

Kwasnica, A.M., Sherstyuk, K., 2007. Collusion and equilibrium selection in auctions, Economic Journal 117(516), 120-45.

Normann, HT., Rau, H.A., 2015. Simultaneous and sequential contributions to step-level public goods: One versus two provision levels. Journal of Conflict Resolution 59(7), 12731300 .

Nosenzo, D., Sefton, M., 2011. Endogenous move structure and voluntary provision of public goods: theory and experiment. Journal of Public Economic Theory 13(5), 721-754.

Potters, J., Sefton, M., Vesterlund, L., 2005. After you - endogenous sequencing in voluntary contribution games. Journal of Public Economics 89(8), 1399-1419.

Sibly, H., Tisdell, J., Evans, S., 2018. Cooperation and turn taking in finitely-repeated prisoners' dilemmas: An experimental analysis. Journal of Economic Psychology 64, 4956.

Van Huyck, J.B., Battalio, R.C., Beil, R.O., 1990. Tacit coordination games, strategic uncertainty, and coordination failure. American Economic Review 80(1), 234-248.

Varian, H.R., 1994. Sequential contributions to public goods. Journal of Public Economics 53(2), 165-186.

Ziegelmeyer, A., Boun My, K., Vergnaud, J., Willinger, M., 2005. Strategic delay and rational imitation in the laboratory. working paper, Max Planck Institute.

Zillante, A., 2011. Recognizing and Playing Turn Taking Strategies. working paper, North Carolina's Urban Research University. 


\section{Table 1}

The joint expected payoffs for any pair of strategies among 21 monotonic strategies and alternating (Wait treatment)

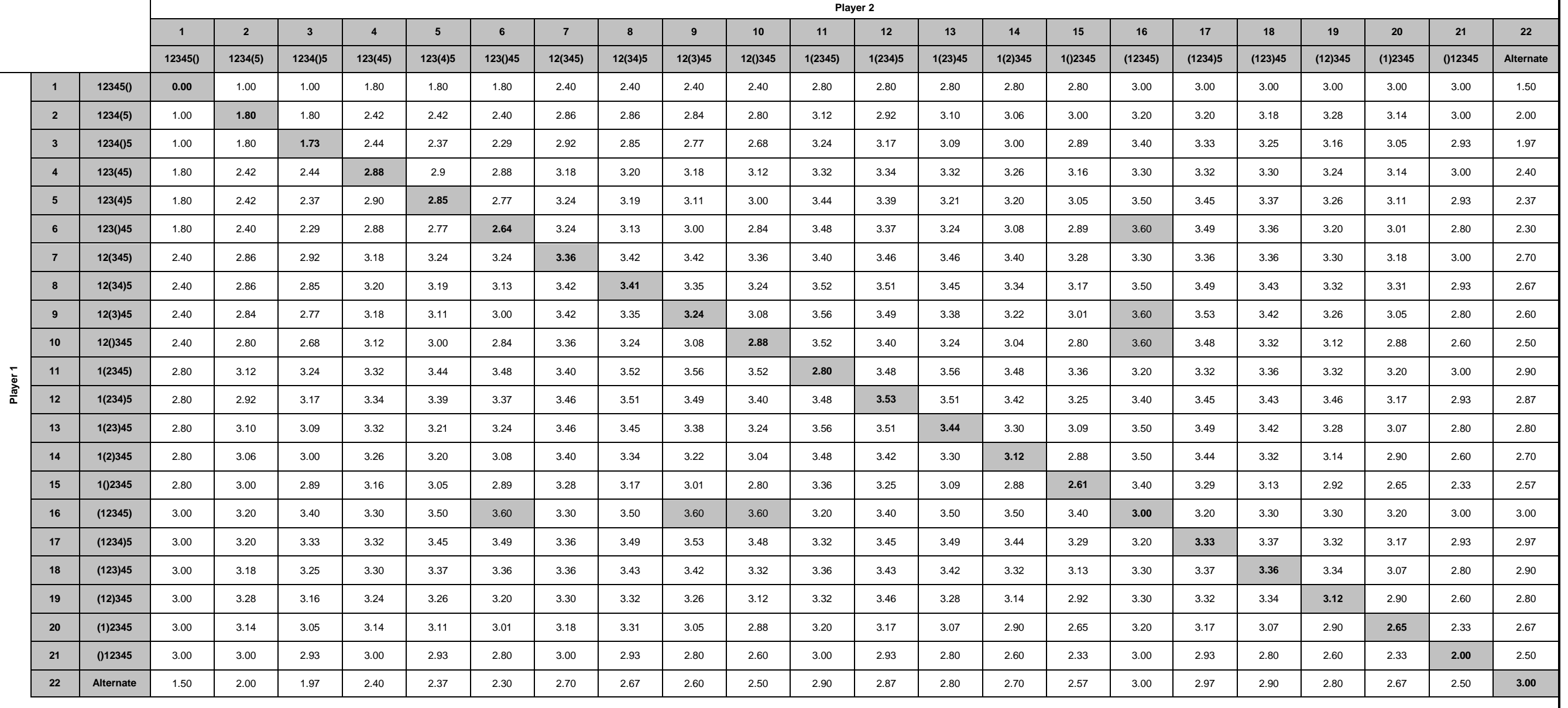

Notation - The player exits on values to the left of the parentheses, waits on values in the parentheses, and enters on values to the right of the parentheses. For example, a player who employs the strategy 12(34)5, exits when he receives a value of 1 or 2, waits on values of 3 and 4 and enters when he receives a 5. 


\begin{tabular}{|l|c|c|c|c|c|c|}
\hline \multicolumn{2}{|l}{ Table 2 - Predictions and Outcomes by treatment } \\
\hline \multirow{2}{*}{ Variable } & \multicolumn{2}{|c|}{ Now } & \multicolumn{2}{c|}{ Wait } & \multicolumn{2}{c|}{ Seq } \\
\cline { 2 - 7 } & Predictions & Outcomes & Predictions & Outcomes & Predictions & Outcomes \\
\hline $\begin{array}{l}\text { payoff-max } \\
\text { cutoff } \\
\text { strategy }\end{array}$ & 12() 345 & $53 / 86$ & $1(234) 5$ & $1 / 88$ & $\begin{array}{c}12() 345- \\
(12345)\end{array}$ & $19.5 / 40$ \\
\hline $\begin{array}{l}\text { (expected) } \\
\text { joint profit - } \\
\text { all rounds }\end{array}$ & 172.8 & 163.2 & 212.0 & 167.9 & 216.0 & 170.2 \\
\hline $\begin{array}{l}\text { efficient } \\
\text { cooperation }\end{array}$ & $48 \%$ & $36.4 \%$ & $80 \%$ & $38.5 \%$ & $88 \%$ & $41.8 \%$ \\
\hline $\begin{array}{l}\text { inefficient } \\
\text { cooperation }\end{array}$ & $0 \%$ & $2.5 \%$ & $12 \%$ & $1.7 \%$ & $12 \%$ & $3.8 \%$ \\
\hline double entry & $36 \%$ & $52.4 \%$ & $4 \%$ & $57.1 \%$ & $0 \%$ & $53.8 \%$ \\
\hline double exit & $16 \%$ & $8.7 \%$ & $4 \%$ & $2.8 \%$ & $0 \%$ & $0.6 \%$ \\
\hline Total & $100.0 \%$ & $100.0 \%$ & $100.0 \%$ & $100.0 \%$ & $100.0 \%$ & $100.0 \%$ \\
\hline
\end{tabular}

Notes: payoff-max cutoff strategy indicates the cutoff strategy that maximizes the pair's joint expected payoff in each treatment (Predictions) and the fraction of subjects that actually played this strategy (Outcomes).

(expected) joint profit - all rounds gives the joint expected profit from playing the above payoff-maximizing cutoff strategy (Predictions) and the mean realized pair payoff over all rounds (Outcomes).

The next four rows give the breakdown of predicted outcomes if the payoff-maximizing strategy is played and the realized breakdown of outcomes by treatment.

Efficient cooperation - only the subject with the (weakly) higher value enters.

Inefficient cooperation - only the subject with the (strictly) lower value enters.

Double entry - both subjects enter.

Double exit - both subjects exit.

\section{Table 3 - Overall Entry Percentages by Treatment and for Stage 1 for Wait and Seq}

Left Panel - frequency of entry for each value for Now, Wait and Seq treatments.

Center Panel - distribution of stage-1 entry, wait and exit decisions for each value in Wait treatment.

Right Panel - frequency of stage-1 entry for each value for Seq treatment.

\begin{tabular}{|c|c|c|c|c|c|c|c|}
\hline & \multicolumn{3}{|c|}{ Overall Entry } & \multicolumn{3}{c|}{ Wait Stage-1 Decision } & Seq Stage-1 \\
Entry
\end{tabular}


Table 4 - Stage-two entry percentages conditional on partner's stage-one decision

\begin{tabular}{|c|c|c|c|c||c|c|c|}
\hline \multirow{2}{*}{ Value } & \multicolumn{4}{|c||}{ Wait } & \multicolumn{3}{c|}{ Seq } \\
\cline { 2 - 8 } & exit & enter & wait & total & exit & enter & total \\
\hline 1 & $97.6 \%$ & $26.8 \%$ & $61.4 \%$ & $50.7 \%$ & $92.6 \%$ & $28.2 \%$ & $49.1 \%$ \\
\hline 2 & $100 \%$ & $30.1 \%$ & $64.0 \%$ & $47.9 \%$ & $95.2 \%$ & $42.3 \%$ & $52.9 \%$ \\
\hline 3 & $100 \%$ & $44.1 \%$ & $92.6 \%$ & $71.3 \%$ & $99.3 \%$ & $81.9 \%$ & $86.7 \%$ \\
\hline 4 & $100 \%$ & $79.7 \%$ & $100 \%$ & $91.5 \%$ & $100 \%$ & $92.1 \%$ & $94.0 \%$ \\
\hline 5 & $100 \%$ & $95.9 \%$ & $100 \%$ & $98.1 \%$ & $100 \%$ & $96.0 \%$ & $96.9 \%$ \\
\hline
\end{tabular}

Table 5 - Summary statistics for pair profits by treatment and, for Seq, by player type

\begin{tabular}{|l|c|c|c|c|c|}
\hline Treatment & Obs. & Mean & Std. Dev. & Min & Max \\
\hline Now & 43 & 163.2 & 13.1 & 129.3 & 181 \\
\hline Wait & 44 & 167.9 & 26.0 & 125.3 & 215.7 \\
\hline Seq & 40 & 170.2 & 23.9 & 128.7 & 211.3 \\
\hline Seq - Player 1 & 40 & 86.9 & 13.8 & 60.7 & 118 \\
\hline Seq - Player 2 & 40 & 83.3 & 13.8 & 58.7 & 106 \\
\hline
\end{tabular}


Table 6 - Best-Fit Strategies in Wait

Distribution of best-fit strategies with the mean fraction of errors by strategy, mean own and partner's profit and mean own and partner's entry frequency for Wait, based on rounds range 6-55

\begin{tabular}{|c|c|c|c|c|c|c|c|}
\hline & \multicolumn{6}{|c|}{ Wait } \\
\hline & Strategy & $\begin{array}{c}\text { Number } \\
\text { who played } \\
\text { strategy }\end{array}$ & $\begin{array}{l}\text { Fraction } \\
\text { of errors }\end{array}$ & $\begin{array}{l}\text { Mean } \\
\text { profit }\end{array}$ & $\begin{array}{c}\text { Mean } \\
\text { profit of } \\
\text { partner }\end{array}$ & $\begin{array}{c}\text { Entry } \\
\text { percent } \\
\text { (after both } \\
\text { stages) }\end{array}$ & $\begin{array}{c}\text { Partner entry } \\
\text { percent (after } \\
\text { both stages) }\end{array}$ \\
\hline \multirow{10}{*}{ 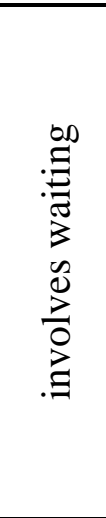 } & 12 (3) 45 & 7 & .07 & 96.0 & 93.4 & 0.63 & 0.66 \\
\hline & $1(2345)$ & 0.5 & .36 & 63.7 & 75.7 & 0.88 & 0.98 \\
\hline & $1(234) 5$ & 1 & .08 & 92.0 & 123.7 & 0.45 & 0.52 \\
\hline & $1(23) 45$ & 24 & .08 & 95.0 & 93.3 & 0.64 & 0.65 \\
\hline & $1(2) 345$ & 1.25 & .26 & 82.7 & 75.4 & 0.81 & 0.79 \\
\hline & (12345) & 5.5 & .26 & 69.0 & 64.5 & 0.98 & 0.90 \\
\hline & (1234) 5 & 1.25 & .43 & 80.6 & 62.1 & 0.97 & 0.77 \\
\hline & (123) 45 & 12.75 & .15 & 82.3 & 79.9 & 0.80 & 0.75 \\
\hline & (12) 345 & 12.5 & .15 & 83.6 & 80.0 & 0.82 & 0.80 \\
\hline & (1) 2345 & 8.5 & .18 & 71.2 & 72.0 & 0.90 & 0.89 \\
\hline \multirow{8}{*}{ 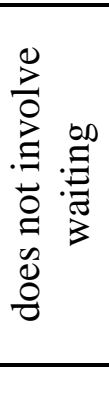 } & () 12345 & 8 & .20 & 67.7 & 68.9 & 0.90 & 0.86 \\
\hline & $1($ ) 2345 & 4.75 & .15 & 74.8 & 80.4 & 0.84 & 0.90 \\
\hline & 12 () 345 & 1 & .26 & 67.0 & 88.3 & 0.77 & 0.90 \\
\hline & 123() 45 & - & - & - & - & & \\
\hline & 1234 () 5 & - & - & - & - & & \\
\hline & 12345() & - & - & - & - & & \\
\hline & alternating & - & - & - & - & & \\
\hline & Average & Total 88 & .14 & 85.2 & 85.2 & 0.76 & 0.76 \\
\hline
\end{tabular}


Table 7 - Best-Fit Strategies in Now and Seq

Distribution of best-fit strategies with the mean fraction of errors by strategy, mean own and partner's profit and mean own and partner's entry frequency for Now and for Player 1 in Seq, based on rounds range 6-55

\begin{tabular}{|c|c|c|c|c|c|c|c|c|c|c|c|c|}
\hline \multirow[b]{2}{*}{ Strategy } & \multicolumn{6}{|c|}{ Now } & \multicolumn{6}{|c|}{ Seq } \\
\hline & $\begin{array}{c}\text { Number } \\
\text { who } \\
\text { played } \\
\text { strategy }\end{array}$ & $\begin{array}{l}\text { Fraction } \\
\text { of errors }\end{array}$ & $\begin{array}{l}\text { Mean } \\
\text { profit }\end{array}$ & $\begin{array}{c}\text { Mean } \\
\text { profit of } \\
\text { partner }\end{array}$ & $\begin{array}{c}\text { Entry } \\
\text { percent }\end{array}$ & $\begin{array}{c}\text { Partner } \\
\text { entry } \\
\text { percent }\end{array}$ & $\begin{array}{c}\text { Number } \\
\text { who played } \\
\text { strategy }\end{array}$ & $\begin{array}{l}\text { Fraction } \\
\text { of errors }\end{array}$ & $\begin{array}{l}\text { Mean } \\
\text { profit }\end{array}$ & $\begin{array}{c}\text { Mean } \\
\text { profit of } \\
\text { partner }\end{array}$ & $\begin{array}{c}\text { Entry } \\
\text { percent }\end{array}$ & $\begin{array}{c}\text { Partner } \\
\text { entry } \\
\text { percent }\end{array}$ \\
\hline () 12345 & 13.5 & 0.11 & 74.0 & 70.3 & 0.89 & 0.85 & 9 & 0.04 & 65.0 & 60.3 & 0.96 & 0.92 \\
\hline 1 () 2345 & 15 & 0.09 & 77.0 & 76.7 & 0.81 & 0.81 & 7 & 0.05 & 75.6 & 70.1 & 0.82 & 0.82 \\
\hline 12 () 345 & 53 & 0.42 & 84.6 & 85.6 & 0.66 & 0.68 & 19.5 & 0.06 & 81.0 & 80.5 & 0.70 & 0.74 \\
\hline 123 () 45 & 0.5 & 0.16 & 77.7 & 87.3 & 0.57 & 0.60 & 4.5 & 0.06 & 96.4 & 88.5 & 0.52 & 0.62 \\
\hline 1234 () 5 & - & - & - & - & - & - & - & - & - & - & - & - \\
\hline 12345() & - & - & - & - & - & - & - & - & - & - & - & - \\
\hline alternating & 4 & 0.14 & 85.3 & 85.3 & 0.56 & 0.56 & - & - & - & - & - & - \\
\hline Average & Total 86 & 0.30 & 81.6 & 81.6 & 0.72 & 0.72 & Total 40 & 0.05 & 78.2 & 75.0 & 0.76 & 0.79 \\
\hline
\end{tabular}


Figure 1 - Histogram of Paired Subjects' Joint Profits

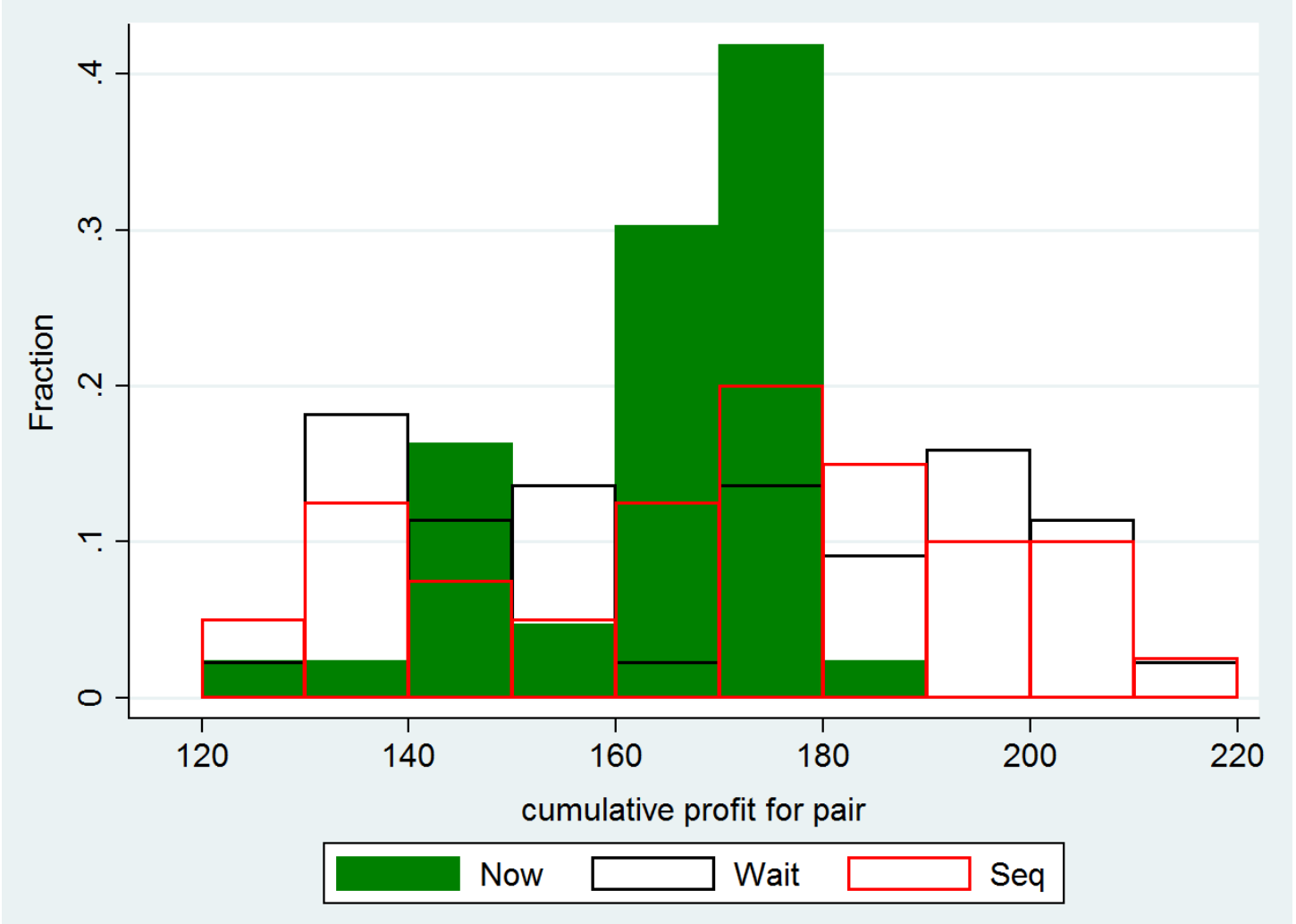

Figure 2 - Stage-Two Entry Frequencies for the Lowest and Highest Earning Pairs

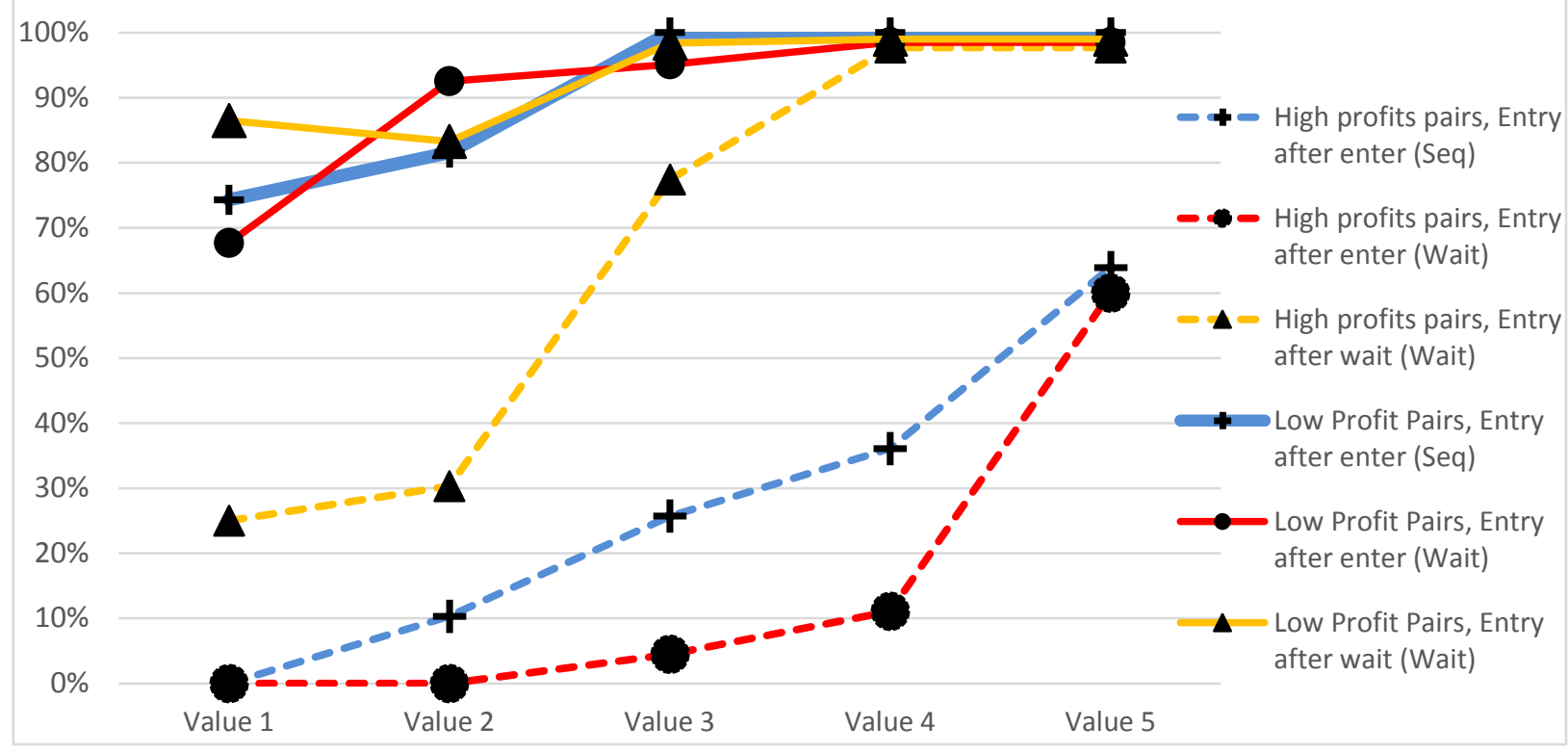




\section{Appendix: Monotonic Strategies}

Proposition: The pair's joint expected profits are maximized by each pair member using a monotonic strategy in the first stage of the two-stage game. As long as the pair's range of possible numbers overlap when the numbers are strictly positive, this will never entail the degenerate case of one player always entering and the other always exiting.

Proof: We will prove this in a more general framework than our simple game. Suppose that each player $i$ receives a randomly drawn integer between $a_{i}$ and $b_{i}$ inclusive where the probability of receiving a number $x$ is $\pi_{x}^{i}$ (where $\pi_{x}^{i}>0$ and $\left.\sum_{x \in\left\{a_{i} \ldots b_{i}\right\}} \pi_{x}^{i}=1\right)$. An overlap is said to exist if $b_{i}>a_{j}$ and $b_{j}>a_{i}$.

By exiting a player receives zero and by entering he receives his number if the other player exits, but receives some function $f(x)$ increasing in his number, $x$, if both enter (in stage 1 or in stage 2). We assume that $f(x)$ is strictly less than his number $x$ (and $f^{\prime}(x)<1$ ); hence entry imposes a negative externality on the other player. We also assume that if it is profitable for a player to enter alone (that is, his value is greater than zero), then it is also profitable for him to enter when his partner enters $(x>f(x)>0, x>0)$.

The cooperative solution is given by the pair of strategies that maximizes the sum of the players' expected payoffs. If a player waited in stage 1 , we assume he will enter and receive his number if his partner exited in stage 1 (and $x>0$ ), and he will exit and receive zero if his partner entered in stage 1 . We note that if his partner entered in stage 1, it may still maximize joint payoffs for him to enter if his value is high enough and $f(x)$ is sufficiently large; however, we assume that in such cases the player would simply have chosen to enter in the first place.

For all cases where both players enter it will yield the same payoffs, but the joint payoffs could be higher if the partner waited since now the partner would know that the player is entering (while this wouldn't be known if the player waits and enters). Suppose the partner enters with probability $p(y)$ and waits with probability $t(y)$ when his number is $y$. The value of both waiting and optimally cooperating in the second stage is denoted by $z(x, y)$. For simplicity, we will write the expressions assuming all numbers in the range are positive. The joint expected payoff to entering in stage 1 with number $x$ is

$$
\operatorname{enter}(x)=\sum_{y} \pi_{y}^{-i}[x(1-p(y)-t(y))+p(y)[f(x)+f(y)]+x \cdot t(y)] .
$$

The joint expected payoff to exiting in stage 1 with number $x$ is

$$
\operatorname{exit}(x)=\sum_{y} \pi_{y}^{-i} \cdot y \cdot[p(y)+t(y)] .
$$

The joint expected payoff to waiting in stage 1 with number $x$ is

$$
\text { wait }(x)=\sum_{y} \cdot \pi_{y}^{-i}[y p(y)+x(1-p(y)-t(y))+t(y) z(x, y)] .
$$


We now show that decisions are monotonic in $x$, namely, that if a player chooses to wait for $x$, then the player would never choose to exit for $x^{\prime}>x$, and if a player chooses to enter for $x$, then the player would never choose to exit or wait for $x^{\prime}>x$. Note that monotonicity in one direction implies monotonicity in the other direction, that is, if a player chooses to exit for $x$, then the player would never choose to wait or enter for $x^{\prime}<x$, and if a player chooses to wait for $\mathrm{x}$, then the player would never choose to enter for $x^{\prime}<x$.

First, notice that $\sum_{y} \pi_{y}^{-i} t(y) z(x, y)$ is weakly increasing in $x$ since if there are two values of $x, x^{\prime}$ and $x^{\prime \prime}$ and $x^{\prime \prime}>x^{\prime}$, then one can duplicate the strategy of $x^{\prime}$ with $x^{\prime \prime}$. Now notice that

$$
\operatorname{wait}(x)-\operatorname{exit}(x)=\sum_{y} \pi_{y}^{-i}[x(1-p(y)-t(y))+t(y) z(x, y)-y \cdot t(y)]
$$

is weakly increasing in $x$ and strictly increasing if $p(y)+t(y)<1$. This latter condition can be interpreted as the other player sometimes exits.

The difference between enter and wait is

$$
\operatorname{enter}(x)-\operatorname{wait}(x)=\sum_{y} \pi_{y}^{-i}[\{p(y)[f(x)+f(y)]-y \cdot(y)+t(y)[x-z(x, y)]\}] .
$$

The first term is strictly increasing in $x$ if $p(y)>0$. We now show that the last term, $\sum_{y} \pi_{y}^{-i} \cdot t(y)[x-z(x, y)]$, is weakly increasing in $x$ (and strictly increasing if the other player sometimes enters after waiting).

The function $z(x, y)$ depends upon the entry decisions of the players and is a maximization of the expected joint surplus (and in particular for each $x$, the entry decisions based upon that $x$ should maximize the expected joint surplus given that value of $x$ and the other player's strategies). Thus, we can invoke the Envelope Theorem. We denote this conditional joint surplus as $g(x):=$ $\sum_{y} \pi_{y}^{-i} \cdot t(y)[z(x, y)]$, and thus we have $g^{\prime}(x)=\sum_{y} \pi_{y}^{-i} \cdot t(y)\left[z_{x}(x, y)\right]$. Denote $e_{x}$ and $e_{y}$ as the probability of entering for the corresponding values of $x$ and $y$. We see that $g^{\prime}(x) \leq \sum_{y} \pi_{y}^{-i} \cdot t(y)$ since $z(x, y)=e_{x}\left(1-e_{y}\right) x+e_{y}\left(1-e_{x}\right) y+e_{x} e_{y}[f(x)+$ $f(y)]$ and $z_{x}(x, y)=e_{x}\left(1-e_{y}\right)+e_{x} e_{y} f^{\prime}(x) \leq e_{x}\left(1-e_{y}\right)+e_{x} e_{y}=e_{x} \leq 1$ since $f^{\prime}(x)<1$. Note that $z_{x}(x, y)$ is strictly less than 1 if $e_{y}>0$. (If $e_{y}>0$ and $e_{x}=0$, we have $z_{x}=0$. If $e_{y}>0$ and $e_{x}>0$, we have $e_{x} e_{y} f^{\prime}(x)<e_{x} e_{y}$ since $f^{\prime}(x)<1$.) Thus, enter $(x)-$ wait $(x)$ is strictly increasing in $x$ if the other player sometimes enters or enters after waiting.

We still have to worry about the case of indifference between waiting and entering for several values of $x$. Indifference occurs only if the partner always stays out. The pair then earns the same whether the player enters or waits and then enters. In a repeated game, one player always exiting and the other always entering (for positive values) can take the form of alternating. Here we prove the second part of the proposition. As long as the upper bound of one player $L$ 's range of numbers strictly exceeds zero and the lower bound of his partner $H$ 's range of numbers (and vice-versa), always exiting and alternating in the repeated game can never be socially optimal since the player always exiting (player $L$ ) can wait with his highest number and the player always entering 
(player $H$ ) can wait with any number strictly below that number. Whenever both players wait, player $L$ enters and player $H$ exits in stage 2 to obtain a higher joint payoff than from player $L$ always exiting. Notice that this argument does not depend upon the function $f(x)$, since it entails replacing a lower number with a higher one.

Similar arguments can be made for the monotonicity between wait and exit. We found wait $(x)$ - exit $(x)$ is strictly increasing in $x$ as long as the other player sometimes exits. Say player $S$ never exits and player $W$ uses a non-monotonic strategy of waiting with $x_{1}$ and exiting for $x_{2}>x_{1}$. The only way that this can be optimal is if player $W$ exits after waiting with $x_{1}$ while player $S$ always enters after waiting. The payoff would be equivalent to a monotonic strategy. We know from the above that player $W$ cannot always be exiting. Thus, an equivalent strategy would be $W$ exiting up to $x^{\prime}$ and entering for all $x^{\prime \prime}>x^{\prime}$ with $S$ always entering. We can see that indeed there can be a non-monotonic strategy that is optimal in the following example. Player $W$ has an equal chance of a number of 1,2 , or 100 and player $S$ always has a number of 100 . (For simplicity, we drop the assumption that $W$ must have a strictly positive chance of receiving the numbers between 3 and 99.) The congestion function is $f(x)=\frac{2}{3} x$. An optimal cooperative strategy is for $S$ to always enter and $W$ to (i) wait with 1 and then exit, (ii) always exit with 2 , and (iii) always enter with 100 . This is equivalent to a monotonic strategy of $S$ always entering and $W$ entering on 100 and exiting on 1 or 2 . We note that if $f(x)<x / 2$ and players' ranges overlap, we cannot generate such examples since it would never be optimal for a player to always enter. 